\title{
Northern Analyses Using Single-Stranded Probes Do Not Support a Role for GATA/GACA Repeats in Sex Determination in Mice and Men
}

\author{
EDWARD J. DURBIN, JOHN R.D. STALVEY, AND ROBERT P. ERICKSON \\ Departments of Human Genetics and Pediatrics, University of Michigan Medical School, Ann Arbor, Michigan
}

\begin{abstract}
The possible role of GATA/ GACA repeated sequences in mammalian sex determination was investigated using Northern analyses of mouse and human RNA. Brain, liver, and gonadal RNA from three developmental stages of mice of both sexes and also human fetal RNA from various tissues were hybridized to both sense and antisense Bkm riboprobes as well as to the synthetic oligonucleotide (GATA) 5 . At low levels of stringency, putative transcripts of various sizes were observed in all tissue samples with all probes. At high stringency, only a putative transcript of approximately $12 \mathrm{~kb}$ was observed, but this was later shown to consist of contaminating DNA. No sex-specific differences were observed in any tissue or developmental stage. Thus, we find no evidence that the GATA/GACA repeated sequences are specifically expressed in quantities detectable by Northern analyses in a manner important to mammalian sex determination.
\end{abstract}

Key Words: Bkm sequences, Gonad differentiation, Riboprobes

\section{INTRODUCTION}

The sex-specific Banded krait minor (Bkm) satellite sequences have attracted considerable attention since they were first reported (Singh et al,, 1976). This satellite DNA cross-hybridizes to DNA from a variety of organisms, from Drosophila to humans, which indicates either sequence conservation or independent evolution (Singh et al., 1980, 1981; Levinson et al., 1985). In addition, the sex-specific hybridization patterns observed in some of these organisms suggested a possible role in sex determination. $\mathrm{Bkm}$ sequences contain many GATA tetranucleotide repeats as well as some GACA repeats (Epplen et al., 1982; Singh et al., 1984). They have been found in open reading frames and if translated could encode a very hydrophobic protein. Singh and coworkers (1984) found Bkm sequences to be transcribed with preferential transcription of the GATA strand in different tissues of mice and Drosophila. In addition, these investigators found tran- scription to be male specific and developmentally regulated. However, the sex specificity of these transcripts has not been confirmed by other investigators (Epplen et al., 1982; Schafer et al., 1986). None of these studies used fetal tissue except liver, which was not distinguished by sex.

If $\mathrm{Bkm}$ sequences are involved in primary sex determination, we postulated that they might be expressed in a sex-specific or tissue-specific pattern nearer to the time of gonadal differentiation. Using both the sense and antisense Bkm riboprobes (Erickson et al., 1987), as well as the synthetic oligonucleotide (GATA) $)_{5}$, we were unable to detect age, sex, or tissue-specific transcripts in these human or mouse samples. From these results, we suggest that there is little evidence that Bkm sequences are transcribed and translated to effect sex determination.

\section{MATERIALS AND METHODS}

Total cellular RNA was isolated from brain, liver, and gonads of 14 day postcoitum, newborn, and adult random bred mice of each sex by the guanidinium isothiocyanate-cesium chloride method (Chirgwin et al., 1979). Total human RNA was isolated using guanidinium- $\mathrm{HCl}$ (Chirgwin et al., 1979) from 13.5 to 21 week fetuses aborted by dilation and extraction in 1984. Since the fetal remnants were fragmented and most tissues unidentifiable, fetal sex was established using the human $\mathrm{Y}$ chromosomal centromeric probe Y97 (Stalvey and Erickson, 1987). Kidneys, lung, heart, and placenta were positively identified tissues, whereas several "glands" might have included gonads. For some samples, poly $(\mathrm{A})^{+}$RNA was isolated from

Received August 2, 1988; accepted October 17, 1988.

Address reprint requests to Robert P. Erickson, Department of Pediatrics, University of Michigan Medical School, Ann Arbor, MI 48109.

John R.D. Stalvey is now at the Department of Biological Sciences, Kent State University, Kent, OH 44242. 
total RNA using one pass over oligo(dT) cellulose (Maniatis et al., 1982).

Approximately $10 \mu \mathrm{m}$ of each total mouse and $5 \mu \mathrm{g}$ of each total human RNA sample was run on $1.5 \%$ agarose-formaldehyde denaturing gels (Maniatis et al., 1982) and transferred to Gene Screen Plus. For Northern hybridization with either the sense or antisense $\mathrm{Bkm}$ riboprobes, filters were prehybridized at $65^{\circ} \mathrm{C}$ in 6 $\times$ SSC, $5 \times$ Denhardt's solution, and $0.5 \%$ SDS. Bkm riboprobes derived from PCS316 (Singh et al., 1981) were synthesized using the pGEM vector system (Promega Biotec, Madison, WI) and either the SP6 (sense) or T7 (antisense) polymerase (Erickson et al., 1987). The probes were labeled with ${ }^{32} \mathrm{P}$-UTP to $1 \times 10^{9}$ $\mathrm{cpm} / \mu \mathrm{g}$ and added to a final concentration of $6 \times 10^{5}$ $\mathrm{cpm} / \mathrm{ml}$ and $50 \mu \mathrm{g} / \mathrm{ml}$ denatured Escherichia coli DNA (which does not contain $\mathrm{Bkm}$ sequences). After hybridization for 16 hours at $65^{\circ} \mathrm{C}$ with constant agitation, filters were washed to $0.1 \times \mathrm{SSC}-0.1 \% \mathrm{SDS}$ at $70^{\circ} \mathrm{C}$ for 30 minutes. Filters were exposed to Kodak XAR film with an intensifying screen at $-70^{\circ} \mathrm{C}$ for up to 10 days before developing.

For hybridization with the synthetic oligonucleotide (GATA $_{5}$, conditions similar to those of Schafer et al. (1986) were used except that the hybridization temperature was $42^{\circ} \mathrm{C}$. This probe was end labeled with $\gamma_{-}{ }^{32} \mathrm{P}-\mathrm{ATP}$ and T4 polynucleotide kinase (Maniatis et al., 1982). Molecular weight determinations were made by isolation of the lane containing DNA size markers and staining it with ethidium bromide or by staining the filters in a solution of $0.04 \%$ methylene blue- $0.5 \mathrm{M}$ sodium acetate to localize $28 \mathrm{~S}$ and $18 \mathrm{~S}$ ribosomal RNAs (Maniatis et al., 1982).

\section{RESULTS}

We used $\mathrm{Bkm}$ riboprobes, both sense and antisense, for Northern hybridizations because RNA-RNA hybrids have a higher Tm than do DNA-RNA or DNA-DNA hybrids and, therefore, are more stable. In addition, very high specific activities can be obtained. When the antisense $\mathrm{Bkm}$ riboprobe (i.e., $5^{\prime} \rightarrow 3^{\prime}$, CUAU) was hybridized to adult mouse total RNA, all tissues of both sexes gave positive signals. At least three different sized signals were observed in all tissues except liver (Fig. 1). One signal was approximately $12 \mathrm{~kb}$ and was observed in all samples. A $4.5-5.0 \mathrm{~kb}$ band and a $1.8-2.0 \mathrm{~kb}$ band were visible in all tissues except in the liver of both males and females. Additional smaller bands $(<1.8 \mathrm{~kb})$ were observed in ovary and male brain RNA samples. The $4.5-5.0 \mathrm{~kb}$ band was present in all tissue RNAs from both sexes of fetal (14 days postcoitum) and newborn mouse samples, whereas the $1.8-2.0 \mathrm{~kb}$ band was present in all but male liver (results not shown).

The sense riboprobe (i.e., $5^{\prime} \rightarrow 3^{\prime}$, GAUA) hybridized only to a $1.8-2.0 \mathrm{~kb}$ band in all fetal and newborn samples (data not shown); whereas in adults this band,

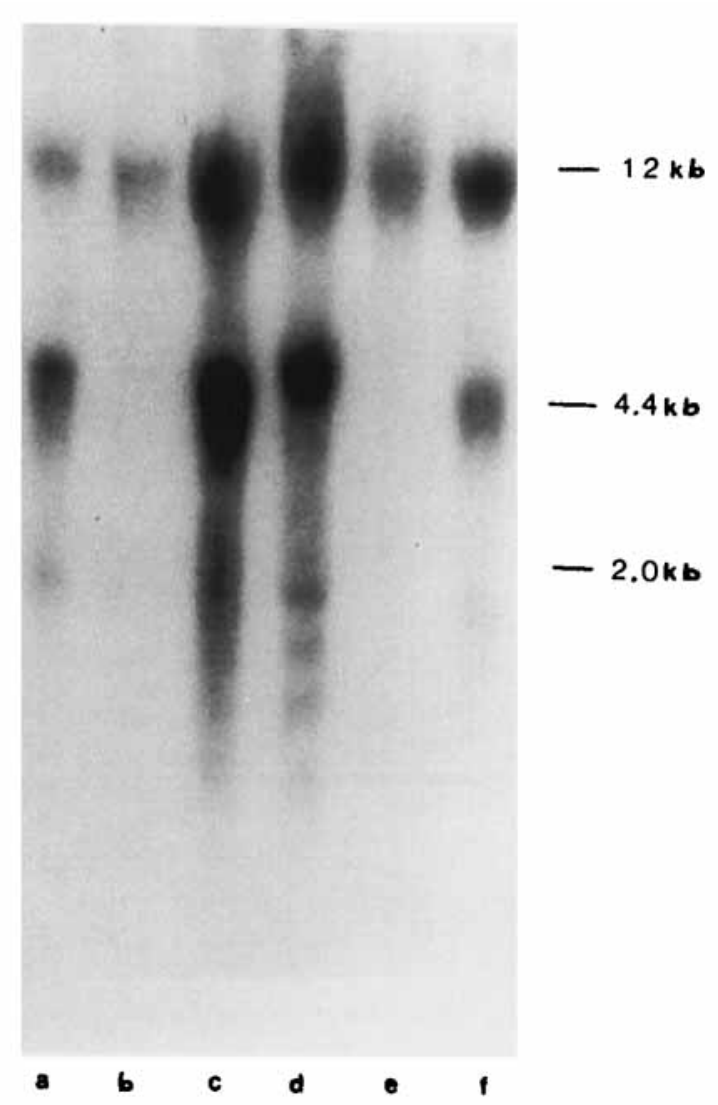

Fig. 1. Northern hybridization of Bkm antisense riboprobe to adult mouse RNA. Results with female (a-c) and male ( $d-f$ ) brain (a,d), liver (b,e), ovary (c), and testes (f) samples are shown.

and also the $12 \mathrm{~kb}$ band, were observed (Fig. 2). All of these results are similar to those of Schafer et al. (1986) who found that both strands of these repeats are expressed differently but no sex differences for each organ were observed. This stands in contrast to the sex-specific differences observed by Singh and coworkers (1984).

Since these hybridizations/washes were carried out at low stringency, they were repeated at high stringency. Under these conditions only the $12 \mathrm{~kb}$ band was observed in all tissues of both sexes (Fig. 3). We were concerned about the possibility that the $1.8-2.0$ and $4.5-5.0 \mathrm{~kb}$ bands were merely nonspecific binding to $18 \mathrm{~S}$ and $28 \mathrm{~S}$ ribosomal RNAs since they are the most abundant species in total RNA preparations. Preparations of $\operatorname{poly}(\mathrm{A})^{+}$RNA were hybridized with the antisense Bkm riboprobe (Fig. 4). All lanes containing total RNA showed all three bands observed previously, but the poly $(A)^{+}$lanes showed only hybridization to $4.5-5.0$ and $1.8-2.0 \mathrm{~kb}$ bands, which probably represent nonspecific hybridization to $28 \mathrm{~S}$ and $18 \mathrm{~S}$ rRNA since contamination with rRNA can occur even after two passes over oligo(dT) columns (Maniatis et al., 


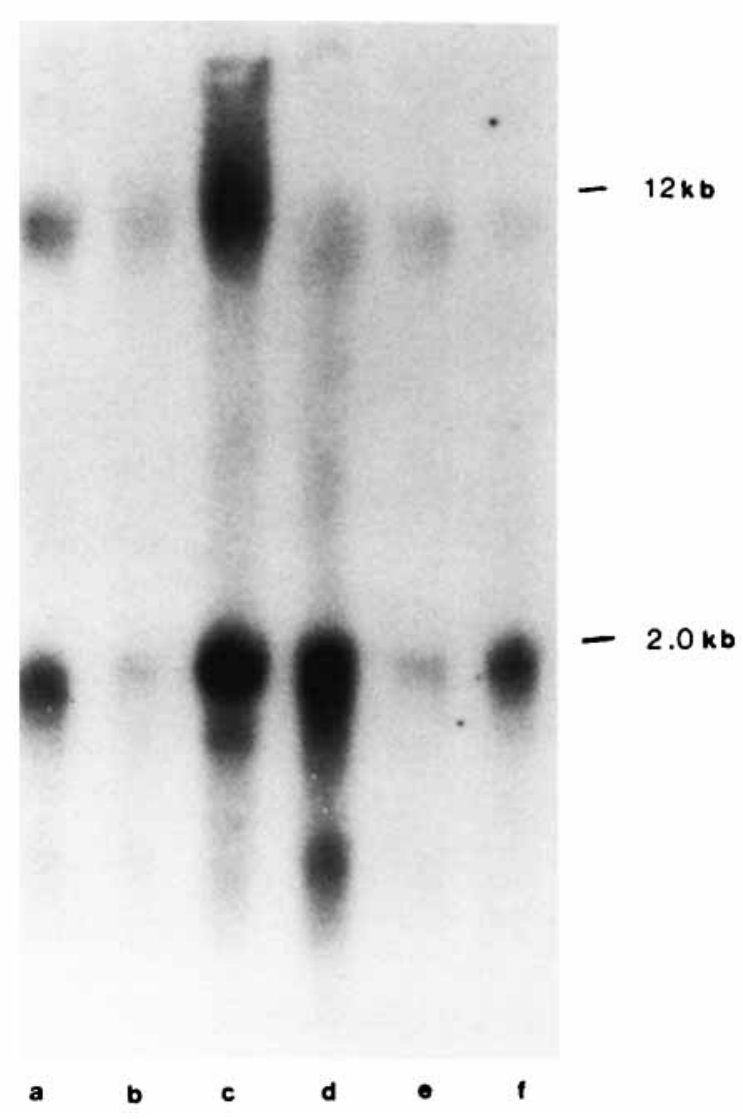

Fig. 2. Northern hybridization of $\mathrm{Bkm}$ sense riboprobe to adult mouse RNA. Results with male (a-c) and female (d-f) testes (a), liver $(b, e)$, brain (c,f), and ovary (d) samples are shown.

1982). The absence of the $12 \mathrm{~kb}$ band in $\operatorname{poly}(\mathrm{A})^{+}$ preparations was puzzling. It was possible that the large "transcript" size prohibited binding to the oligo(dT) cellulose column or that there was DNA present in the total RNA preparations that would not bind to the oligo(dT) column. The possibility of DNA contamination was explored. We treated RNA samples with RNase and observed a band at about $12 \mathrm{~kb}$ (results not shown). We also treated RNA samples with RNase-free DNase and observed no hybridization at the $12 \mathrm{~kb}$ putative transcript size (results not shown). Therefore, we conclude that small amounts of DNA contamination running at an unresolved, about $12 \mathrm{~kb}$ mobility hybridized to our probe since repetitive Bkm sequences in DNA will hybridize strongly. Thus we were unable to see any age-, sex-, or tissue-specific expression of Bkm sequences in mouse samples.

The use of the same sense and antisense Bkm riboprobes for Northern hybridizations to human fetal total RNA gave similar results. Variable degrees of degradation of these difficult to obtain RNAs had occurred so the ethidium bromide-stained gel is illustrated in Figure $5 \mathrm{C}$ for relative quantitation of

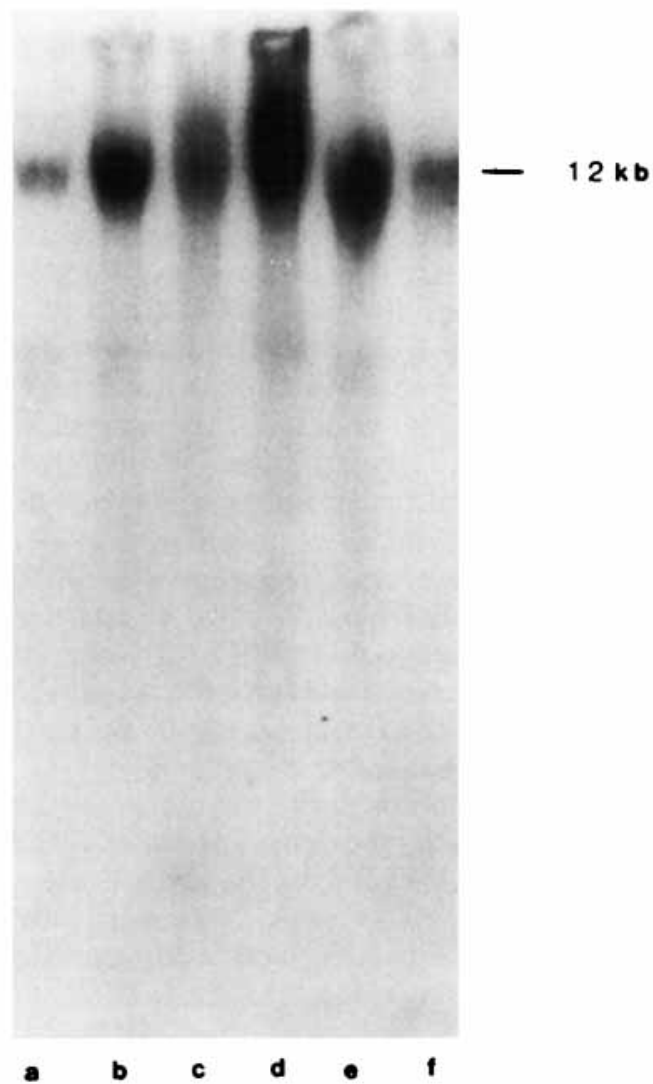

Fig. 3. Northern hybridization of Bkm antisense riboprobe to adult mouse RNA under stringent conditions. Results with female $(a-c)$ and male (d-f) brain (a,d), liver (b,e), ovary (c), and testes (f) samples are shown.

amounts of RNA. With the antisense Bkm riboprobe, hybridization was at $4.5-5.0 \mathrm{~kb}$ in all samples except total fetal or 21 week female "gland" (Fig. 5A). These two lanes had lower amounts of RNA loaded than the other samples. The sense Bkm riboprobe hybridized strongly to a $1.8-2.0 \mathrm{~kb}$ band in all samples except total fetal RNA (Fig. 5B). In addition, fainter hybridization was observed at $4.5-5.0 \mathrm{~kb}$ in most tissues except total fetal RNA, 21 week female "gland," and another unidentified female tissue. These sizes again appear to correspond to the size of $18 \mathrm{~S}$ and $28 \mathrm{~S}$ ribosomal RNAs (Fig. 5C).

Hybridizations with the synthetic oligonucleotide $(\text { GATA })_{5}$ gave results very similar to those observed with the antisense probe under stringent conditions (results not shown). The only hybridization was in the region of approximately $12 \mathrm{~kb}$.

\section{DISCUSSION}

The role of Bkm sequence transcripts in sex determination appears to be minimal in mice and humans. 


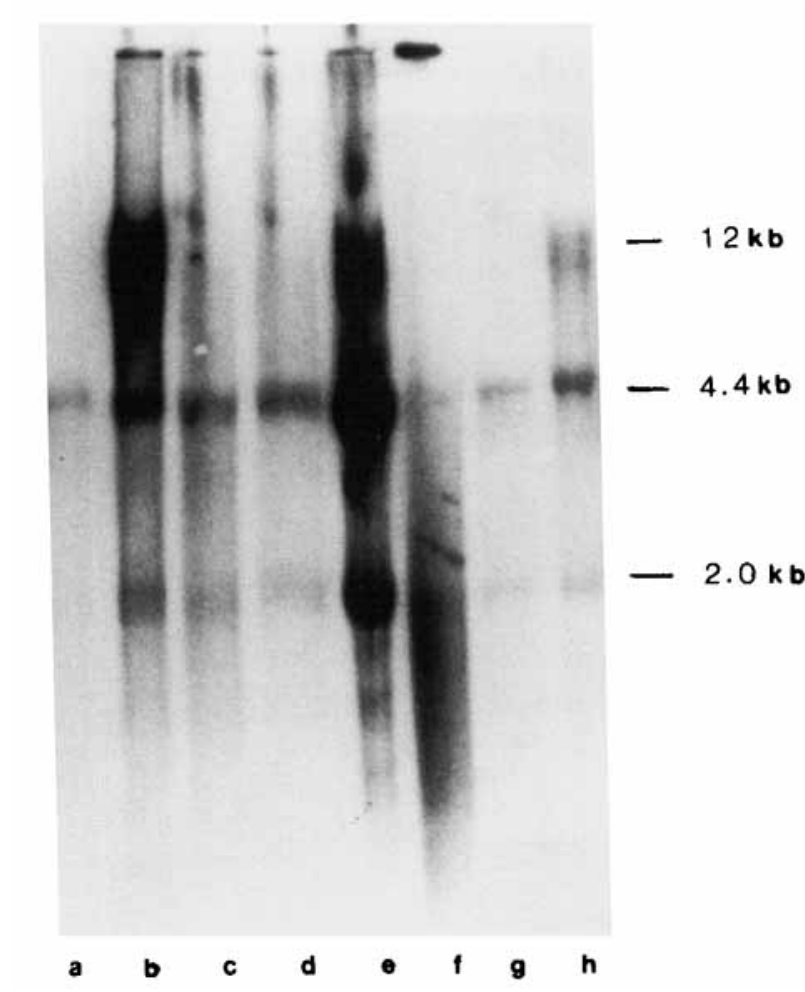

Fig. 4. Bkm antisense riboprobe to various mouse RNA samples. Results with liver poly $\left(\mathrm{A}^{+}\right)$RNA (a,g), total mouse RNA (b,h), adult testes poly $\left(\mathrm{A}^{+}\right)$RNA (c,f), immature testes poly $\left(\mathrm{A}^{+}\right)$RNA (d), immature testes total RNA (e) are shown.

Although others have found transcripts in mice (see Introduction), we found no evidence using sensitive $\mathrm{Bkm}$ riboprobes at moderate to high stringency that either the sense or antisense sequences are expressed in human or mouse tissues. Singh and coworkers (1984) found sex-specific expression of Bkm-related sequences in mice. Using a Drosophila Bkm-containing clone as probe, three distinct mRNA bands were observed in adult male liver but not unsexed embryonic liver. However, the sex- and stage-specific expression has not been confirmed by others. Schafer et al. (1986) performed RNA hybridizations with adult mouse tissues using the synthetic oligonucleotides (GATA) $(\mathrm{CTAT})_{5}$, and $(\mathrm{GACA})_{4}$ as probes. They found three distinct transcripts in total RNA from all tissues in hybridizations with (GATA) $)_{4}$. The transcripts were $1.7 \mathrm{~kb}, 2.4 \mathrm{~kb}$, and $3.0 \mathrm{~kb}$ in size. No sex specificity was observed. Their hybridization patterns and the size of at least one of these transcripts were similar to what we observed at low stringency in all tissues of both sexes for all stages studied with the antisense Bkm probe. However, at high stringency we were unable to detect any transcripts of this size.

Similarly we also were unable to find any specific transcripts at moderate to high stringency using the sense Bkm riboprobe. This result was not surprising, since this probe would hybridize to the antisense or noncoding sequence. However, Schafer et al. (1986) found both sense and antisense Bkm repeat sequences expressed in their studies. In addition, Adelman et al. (1987) reported two mammalian genes transcribed from opposite strands of the same locus in the rat. This would argue for the possibility that the two $\mathrm{Bkm}$ strands are both coding for some function although the sequences for $\mathrm{Bkm}$ make this very unlikely: Translation of the (GATA) strand is not possible because stop codons occur repeatedly in each reading frame. Translation of the other (CTAT) strand would yield an extremely hydrophobic protein. In fact, expression seems very unlikely for this protein, since the synthetic dodecapeptide was soluble only in 50\% formamide (Banting, Goodfellow, and Erickson, unpublished data). Since we cannot find any evidence that these sequences are expressed, it is possible that they function in some other capacity.

One possible function would be that the $\mathrm{Bkm}$ sequences serve as a control element, either expressed (a control RNA) or unexpressed (possibly as identifier sequences as Sutcliffe et al. [1982, 1984] reported for rat brain mRNA). Bkm identifier sequences would function in regulation of the genes involved in sex determination. The validity of the concept of identifier sequences has been challenged; two reports have presented evidence against such identifier sequences (Minarovits et al., 1984; Owens et al., 1985). However, Watson and Sutcliffe (1987) recently found expression of selected repetitive families of DNA to be conserved in monkey and human brain similar to the identifier sequences of rat brain.

Unexpressed Bkm sequences could also function in regulation in the model proposed by Chandra (1985). In this model the main element would be a multicopy, noncoding sequence, which could be Bkm. This sequence, probably Y chromosomal, would function to bind an autosomal repressor preferentially that otherwise would bind to a testis-determining gene on the $\mathrm{X}$ chromosome. The presence of $\mathrm{Bkm}$ would allow the male-determining pathway to proceed. However, evidence that $\mathrm{Bkm}$ sequences bind a protein is lacking, and Page et al. (1987) have cloned the testisdetermining factor from the human $\mathrm{Y}$ and find it to be a member of the zinc finger, presumably regulatory, class. Recently Waring and Pollack (1987) reported the cloning and characterization of dispersed repeated DNA sequences concentrated on the Drosophila X chromosome that are postulated to play a role in dosage compensation. Proof for the function of these repeats is lacking, but the noncoding nature of the sequence, its sequence conservation at dispersed sites on the $\mathrm{X}$, and unichromosomal location share similarities with Chandra's model (1985). However, the sequence conservation breaks down across species of Drosophila (G. 

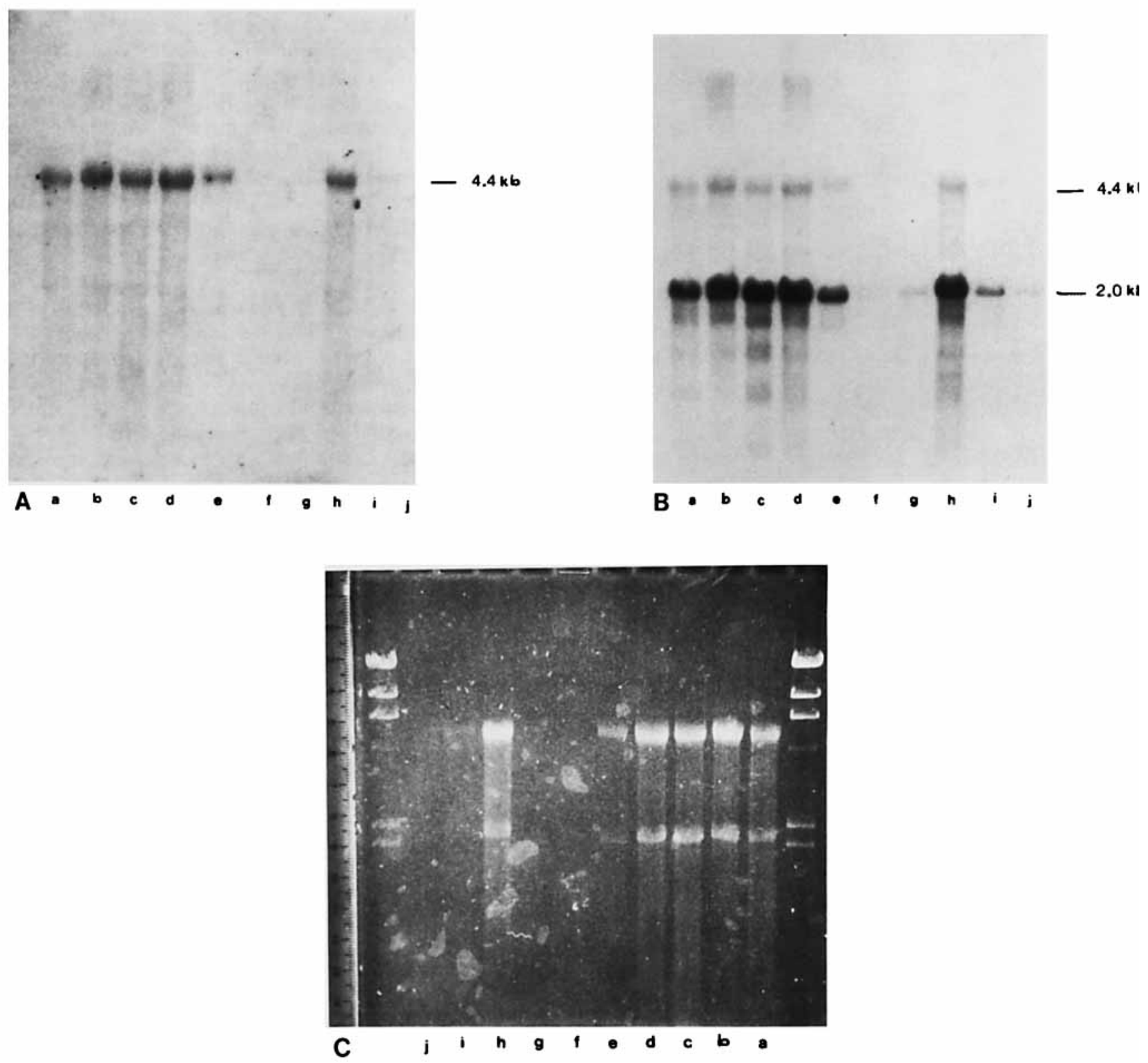

Fig. 5. Bkm riboprobe hybridization to human fetal RNA. A Antisense riboprobe. B: Sense riboprobe. C: Ethidium bromide stained. Results are from "gland" from XY, 13.5 week fetus (a); kidneys from 13.5 week $\mathrm{XY}$ and 15 and 21 week XX fetuses (b,d,h); "gland" from XX, 15 week fetus (c); lung from 15 and 21 week XX fetuses (e,i); total 16 week XY fetus (f); "gland" from XX, 21 week fetus (g); and another "gland" from XX fetus (j).
Waring, personal communication), so its importance may be overstated.

Singh and Jones (1982) believed that Bkm sequences were probably more related to coordinate control than to a direct role in sex determination. They proposed that, at least in snakes, $\mathrm{Bkm}$ sequences most likely functioned in the cycle of condensation of the sex chromosomes. However, this is not true for the mealworm moth Ephestia kuehniella (Traut, 1987). This moth, although having sex chromosomes of the WZ type, contains Bkm sequences only on its autosomes. This autosomal localization of $\mathrm{Bkm}$ sequences does not rule out a relationship with sex determination, however, since the sequences could perform similar functions or could be marking other autosomal loci involved in the sex-determination pathway. The association of $\mathrm{Bkm}$ with mouse proximal chromosome 17 provides evidence for this. Through in situ chromosomal hybrid- 
ization, Bkm sequences have been found to be present in an area of chromosome 17 related to sex determination (Kiel-Metzger and Erickson, 1984). Two deletions in this region of chromosome 17 cause hermaphroditism or sex reversal in mice on a C57BL/6 and Y Dom strain background (Eicher and Washburn, 1986). The possibility that these deletions delete $\mathrm{Bkm}$ sequences has been studied using in situ chromosomal hybridization. However, it appears that at least for one of the deletions, $T^{\mathrm{hp}}$, the deletion does not significantly delete Bkm sequences (Durbin et al., 1988).

The question remains as to whether or not $\mathrm{Bkm}$ sequences serve any function. Perhaps they are merely nonfunctional accumulations on the $\mathrm{Y}$ chromosome. Phillips and coworkers (1982) found this for retroviral sequences on the mouse $\mathrm{Y}$ chromosome. This raises a point regarding the evolutionary conservation of these sequences. Perhaps, as Levinson et al. (1985) propose, the sequences are not conserved but have evolved independently in the wide variety of organisms studied. Differences have been found in the number and sequence of $\mathrm{Bkm}$ repeats in a number of organisms (Epplen et al., 1982; Singh et al., 1984; Erickson et al., 1987). These differences are not incompatible with independent evolution of the $\mathrm{Bkm}$ repeats. However, the role of $\mathrm{Bkm}$ repeat sequences in sex determination, if any, remains unknown.

\section{ACKNOWLEDGMENTS}

We thank Arturo Bevilacqua and Dr. Virginia Hieber for assistance with the Bkm riboprobe construction and Drs. Jerome Gorski and Vera Verga for critical readings of this manuscript. In addition, we thank Mrs. Ann Mogan for typing the manuscript. This work was supported by NIH grants HD20670 and training grant T32 HD07274.

\section{REFERENCES}

Adelman JP, Bond CT, Douglass J, Herbert E (1987): Two mammalian genes transcribed from opposite strands of the same DNA locus. Science 235:1514-1517.

Chandra HS (1985): Sex determination: A hypothesis based on noncoding DNA. Proc Natl Acad Sci USA 82:1165-1169.

Chirgwin JM, Przybla AE, MacDonald RJ, Rutter WJ (1979): Isolation of biologically active ribonucleic acid from sources enriched in ribonuclease. Biochemistry 18:5294-5299.

Durbin EJ, Erickson RP, Craig A (1988): Characterization of GATA/ GACA sequences on mouse chromosome 17. Chromosoma (in press).

Eicher EM, Washburn LL (1986): Genetic control of primary sex determination in mice. Annu Rev Genet 20:327-360.

Epplen J'T, McCarrey JR, Sutou S, Ohno S (1982): Base sequence of a cloned snake W-chromosome DNA fragment and identification of a male-specific putative mRNA in the mouse. Proc Natl Acad Sci USA 79:3798-3802.

Erickson RP, Bevilacqua A, Ross C, Donaldson S, Stalvey JRD (1987): Do Bkm sequences play a role in human sex determination? In FP Haseltine, ME McClure, EH Goldberg (eds): "Genetic Markers of Sex Differentiation." New York: Plenum Press, pp 149-159.

Kiel-Metzger K, Erickson RP (1984): Regional localization of sexspecific Bkm-related sequences on proximal chromosome 17 in mice. Nature 310:579-581.

Levinson G, Marsh JL, Epplen JT (1985): Cross-hybridizing snake satellite, Drosophila, and mouse DNA sequences may have arisen independently. Mol Biol Evol 2:494-504.

Maniatis T, Fritsch EF, Sambrook J (1982): "Molecular Cloning: A Laboratory Manual." Cold Spring Harbor, NY: Cold Spring Harbor Laboratory.

Minarovits J, Kovacs Z, Foldes I (1984): Homologies between a brain-specific identifier (ID) sequence and regions of Harvey murine sarcoma virus and Rous sarcoma virus genomes. FEBS Lett $174: 208-210$.

Owens GP, Chandhari N, Hahn WE (1985): Brain "identifier sequence" is not restricted to brain: Similar abundance in nuclear RNA of other organs. Science 229:1263-1265.

Page DC, Mosher R, Simpson EM, Fisher EMC, Mardon G, Pollack J, McGillivary B, de la Chapelle A, Brown LG (1987): The sexdetermining region of the human $\mathrm{Y}$ chromosome encodes a finger protein. Cell 51:1091-1104.

Phillips SJ, Birkenmeier EH, Callahan R, Eicher EM (1982): Male and female mouse DNAs can be discriminated using retroviral probes. Nature 297:241-243.

Schafer R, Ali S, Epplen JT (1986): The organization of the evolutionary conserved GATA/GACA repeats in the mouse genome. Chromosoma 93:502-510.

Singh L, Jones KW (1982): Sex-reversal in the mouse (Mus musculus) is caused by a recurrent nonreciprocal crossover involving the $\mathrm{X}$ and an aberrant $\mathrm{Y}$ chromosome. Cell 28:205-216.

Singh L, Phillips C, Jones KW (1984): The conserved nucleotide sequences of Bkm, which define $S x r$ in the mouse, are transcribed. Cell 36:111-120.

Singh L, Purdom IF, Jones KW (1976): Satellite DNA and evolution of sex chromosomes. Chromosoma 59:43-62.

Singh L, Purdom IF, Jones KW (1980): Sex-chromosome-associated satellite DNA: Evolution and conservation. Chromosoma 79: $137-157$

Singh L, Purdom IF, Jones KW (1981): Conserved sexchromosome-associated nucleotide sequences in eukaryotes. Cold Spring Harbor Symp Quant Biol 45:805-813.

Stalvey JRD, Erickson RP (1987): An improved method for detecting $Y$ chromosomal DNA. Hum Genet 76:240-243.

Sutcliffe JG, Milner RJ, Bloom FE, Lerner RA (1982): Common 82-nucleotide sequence unique to brain RNA. Proc Natl Acad Sci USA 79:4942-4946.

Sutcliffe JG, Milner RJ, Gottesfeld JM, Lerner RA (1984): Identifier sequences are transcribed specifically in brain. Nature 308: $237-241$.

Traut W (1987): Hypervariable Bkm DNA loci in a moth, Ephestia kuehniella: Does transposition cause restriction fragment length polymorphism? Genetics 115:493-498.

Waring GL, Pollack JC (1987): Cloning and characterization of a dispersed, multicopy, $\mathrm{X}$ chromosome sequence in Drosophila melanogaster. Proc Natl Acad Sci USA 84:2843-2847.

Watson JB, Sutcliffe JG (1987): Primate brain-specific cytoplasmic transcript of the Alu repeat family. Mol Cell Biol 7:3324-3327. 